SAND94-3130

Distribution

Unlimited Release

Printed February 1995

Category UC-700

\title{
The Sandia Total-Dose Estimator: SANDOSE \\ Description and User Guide
}

\author{
C. David Turner \\ Radiation and Electromagnetic Analysis Department \\ Sandia National Laboratories \\ Albuquerque, NM 87185
}

\begin{abstract}
The SANdia total-DOSe Estimator (SANDOSE) is used to estimate total radiation dose to a BRL-CAD solid model. SANDOSE uses the mass-sectoring technique to sample the model using ray-tracing techniques. The code is integrated directly into the BRL-CAD solid model editor and is operated using a simple graphical user interface. Several diagnostic tools are available to allow the user to analyze the results. Based on limited validation using several benchmark problems, results can be expected to fall between a 10\% underestimate and a factor of 2 overestimate of the actual dose predicted by rigorous radiation transport techniques. However, other situations may be encountered where the results might fall outside of this range. The code is written in $\mathrm{C}$ and uses $\mathrm{X}$-windows graphics. It presently runs on SUN SPARCstations, but in theory could be ported to any workstation with a $\mathrm{C}$ compiler and $\mathrm{X}$-windows. SANDOSE is available via license by contacting either the Sandia National Laboratories Technology Transfer Center or the author.
\end{abstract}




\section{Acknowledgments}

Appreciation is given to G. J. Scrivner and S. B. Roeske for many useful discussions during the development of SANDOSE. The encouragement of J. H. Renken is also appreciated with regard to licensing this software. The contribution of M. R. Ackermann in providing an improved ray-distribution algorithm is also recognized. 


\section{DISCLAIMER}

This report was prepared as an account of work sponsored by an agency of the United States Government. Neither the United States Government nor any agency thereof, nor any of their employees, make any warranty, express or implied, or assumes any legal liability or responsibility for the accuracy, completeness, or usefulness of any information, apparatus, product, or process disclosed, or represents that its use would not infringe privately owned rights. Reference herein to any specific commercial product, process, or service by trade name, trademark, manufacturer, or otherwise does not necessarily constitute or imply its endorsement, recommendation, or favoring by the United States Government or any agency thereof. The views and opinions of authors expressed herein do not necessarily state or reflect those of the United States Government or any agency thereof. 


\section{DISCLAIMER}

Portions of this document may be illegible in electronic image products. Images are produced from the best available original document. 


\section{Contents}

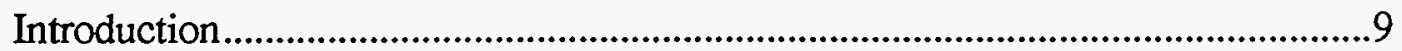

Modeling with MGED for use with SANDOSE.............................................10

General Modeling Practice ....................................................................10

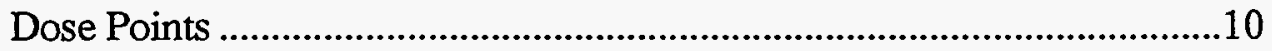

Modeling Detail .........................................................................................11

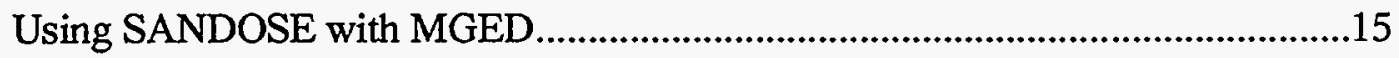

Displaying and Analyzing the Model .....................................................15

Kernels and Kernel Files.........................................................................15

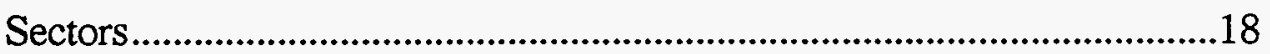

Dose Point Locations, Dose Output File, and Units .................................19

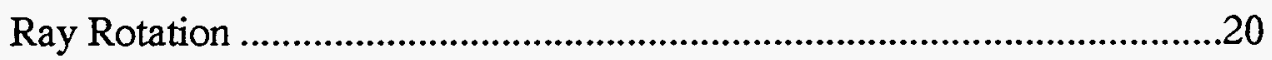

Min Path Approximation ......................................................................20

Graphical Display of Dose Contribution per Sector ..................................20

Areal Density Bins ................................................................................21

Refresh, Dose, and Quit..........................................................................21

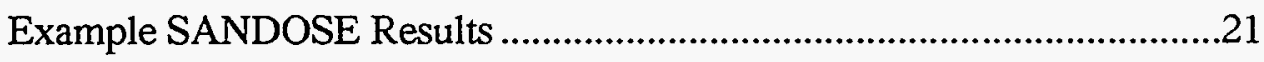

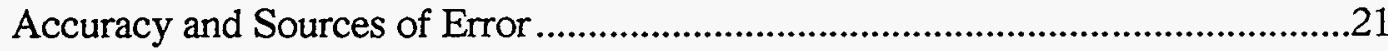

General Accuracy Requirements ...........................................................21

SANDOSE Validation...........................................................................23

Additional Sources of Error ....................................................................26

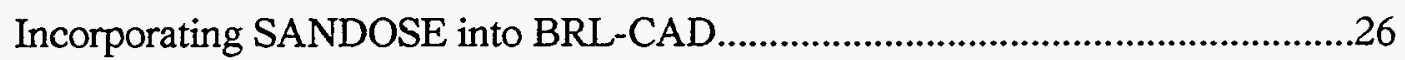

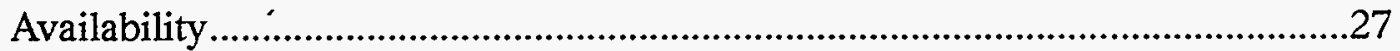

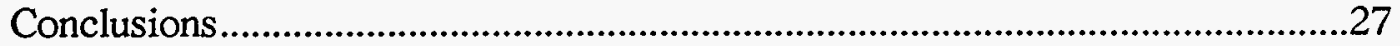

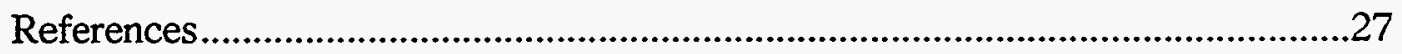




\section{Figures}

Figure 1. MGED model of GPS Block IIR................................................13

Figure 2. MGED model of single GPS subsystem. .....................................13

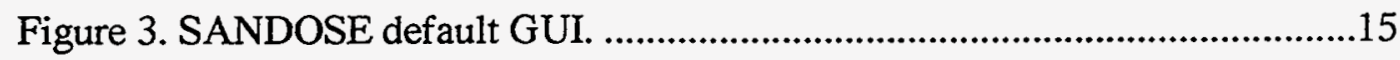

Figure 4. Example user.ker file for SANDOSE. .............................................

Figure 5. Example Point Data File for SANDOSE.........................................19

Figure 6. Example SANDOSE results for subsystem in Figure 2.....................23

Figure 7. Exact SANDOSE results for Board GPR1, Sides1 and 2. ...................25 


\section{Table}

Table 1. Components of the SANDOSE GUI...................................................17 
(This page intentionally left blank) 


\section{The Sandia Total-Dose Estimator: SANDOSE \\ Description and User Guide}

\section{Introduction}

The SANdia total-DOSe Estimator (SANDOSE) is an optional module for the Ballistic Research Laboratory CAD (BRL-CAD) package, Release 4.0 [1]. BRL-CAD is a moderately-advanced, primitive-based, solid-modeling package which is available free of charge (including source code) from the Ballistic Research Laboratory. The purpose of SANDOSE is to estimate total radiation dose at specific locations in a BRL-CAD model. SANDOSE uses the well-known mass-sectoring technique [2,3] to sample the model, using ray tracing techniques, over $4 \pi$ steradians around the dose point of interest, thus assuming omnidirectional radiation. The dose contribution from each sector is found from a 1-Dimensional dose vs. depth curve (kernel), generated by rigorous radiation-transport techniques on a series of solid aluminum spheres for the given radiation environment. The depth for each sector is computed by summing all areal densities encountered by a ray through that sector. The code computes the ray sampling spatial distribution such that all sectors subtend equal solid angle and therefore contribute equally to the total dose.

Although mass-sectoring ignores much of the scattering physics involved in rigorous radiation transport calculations, the technique does provide reasonable results for very complex geometries which would be difficult to model using presently available 3-Dimensional (3-D) radiation-transport techniques. The quality of the results to be expected from SANDOSE is discussed in the validation section.

SANDOSE provides no capability for computing the kernel for a specific environment. All presently-available kernels for SANDOSE were computed using the adjoint mode of the CEPXS/ONELD coupled electron-photon transport code [4]. The naturalelectron kernel, the enhanced-electron kernel, and the total kernel (natural + enhanced + solar flare proton) for GPS Block IIR orbit are included with the software. The user may also specify a user-defined kemel.

A simple graphical user interface (GUI) is used to control the SANDOSE module interactively from within the BRL-CAD Multiple-Device Graphics Editor (MGED). SANDOSE provides several diagnostic tools to evaluate the total-dose results at an individual dose point. These include graphical display of the relative dose contribution per sector and dose bins, each of which can aid the user in determining where most of the existing shadow shielding comes from or where to add additional shielding. 
SANDOSE presently runs on Sun SPARCstations under SunOS 4.1.x and OpenWindows (X-windows) graphics. The code is written in $\mathrm{C}$ and includes approximately 1600 lines in the basic core module, including many comments. The GUI is based on Sun's OpenLook Developer's Guide and XVIEW tookit, which contains widgets for buttons, etc., and adds about 800 additional lines of $\mathrm{C}$ code.

\section{Modeling with MGED for use with SANDOSE}

\section{General Modeling Practice}

Creation of solid models with MGED can be approached in a variety of ways. A large model is made more tractable if a tree is developed with branches and sub-branches flowing out, representing assemblies and sub-assemblies. These assemblies are created using regions or combinations of other assemblies and/or solid primitives. Primitives typically appear only at the lowest levels, i.e., they are the leaves of the tree. A solid in MGED generally refers to an individual primitive, such as a sphere or box, which may or may not be a part of a higher level region or combination.

When creating models for use with SANDOSE, the mass density, in $\mathrm{g} / \mathrm{cc}$, must be provided for all parts. This is done using the MGED mater command. This command sets parameters for regions only, not for solid primitives, so parts which are to be assigned densities must be members of a region. Parts which have the same density can be combined in one region with the density assigned only once for the entire region. The "mater" parameter of interest here is the Parameter string. This string is where SANDOSE extracts the density of the material intersected by a particular ray. This parameter string is entered in the following manner: den $=0.5$ (the leading 0 is important), or $d e n=2.2$. This format is recognized by SANDOSE and the code will extract the proper value. If the user forgets to provide a density, a warning is issued every time a ray encounters a material without a specified density. At present, the calculation will continue with the density set to that of aluminum, 2.7 .

A general modeling rule for SANDOSE which has been followed with success is the following. Primitives which form the lowest-level parts with different materials (i.e., different densities) are placed in regions ( $r$ command in MGED). These parts are then given their appropriate densities using the mater command, as described above. No additional materials are defined above this level in the model. These regions, with their densities defined, are then combined for ease in understanding the model structure into combinations (comb command in MGED). If desired, these combinations can be further combined into higher level combinations using the comb command. Eventually, the model tree is completed with a final combination with which the entire model can be accessed.

\section{Dose Points}

An unfortunate requirement of BRL-CAD is that in order for a ray trace to function properly, the starting point of a ray must be in a void region. This is not a problem for SANDOSE if the dose point of interest is not "buried" within some material or part. How- 
ever, if it is desired to place a dose point within a material, a small spherical void (or another shape) must be placed at that point. Spherical voids, with a radius of about 5 mils and the dose point at the center, have produced good results with SANDOSE.

Probably the most tedious part of preparing to apply SANDOSE to a particular model is the location of the dose points. The cartesian coordinates of each dose point must be provided with respect to the coordinate system used to build the model. The generation of these dose-point coordinates is mostly a manual operation by the user, with some assistance from MGED. Shortcuts can be devised if dose points are arranged in regular arrays, such as on a series of parallel circuit boards. SANDOSE can either read these dose point locations $(x, y, z)$ from a data file or they can be supplied individually. Experience has shown it easier to create a data file of dose points at the outset. These points can either be referenced by number in the SANDOSE GUI or the entire set of dose points can be computed as a whole.

\section{Modeling Detail}

The exact form of the model created with MGED for SANDOSE can be very complex, rendering every detail of the system, or it can be "homogenized" to some extent to simplify the model creation effort. Fortunately, the nature of the mass-sectoring algorithm allows an homogenized model to be used in most cases. The areas in the near vicinity of the dose point are the most important to model accurately. Neighboring boards and box walls contribute much more to the shadow shielding than does any component far on the other side of the spacecraft. For this reason, normal procedure has been to create rather detailed, but still inexact, models of the box containing the system of interest, including primarily generic circuit boards and occasionally additional detail. The weights of these circuit boards and their composition are typically adjusted to provide a good approximation to a "well populated" board. If the board is not fully populated, adjustments can be made by either adjusting the density associated with the board components or actually changing the "component" model(s). Other systems on the spacecraft are modeled by a homogeneous mass of the correct physical size, but with an artificial density to produce the correct total weight. A few numerical experiments with SANDOSE will reveal that the detail included in neighboring boxes and especially those far away is relatively unimportant for the computation of total dose within the box of interest. Including great detail such as device leads or individual components, unless exceptionally heavy, is probably not worthwhile considering the approximations being made in the mass-sectoring technique compared to rigorous radiation-transport calculations.

Figure 1 shows an MGED model of GPS Block IIR which has been used to estimate total dose to several specific subsystems. The boxes shown in color are the subsystems of interest. These boxes have internal detail modeled, such as circuit boards, bulkheads, etc. The other boxes are homogeneous masses to represent the shadow shielding provided by the remainder of the spacecraft. Figure 2 shows part of the internal model for the subsystem shown in yellow, showing the presence of generic homogeneous circuit boards. What appear to be dots on the boards are actually small hemispherical covers over the dose points. These generic circuit board models are described in a later section. 
(This page intentionally left blank) 


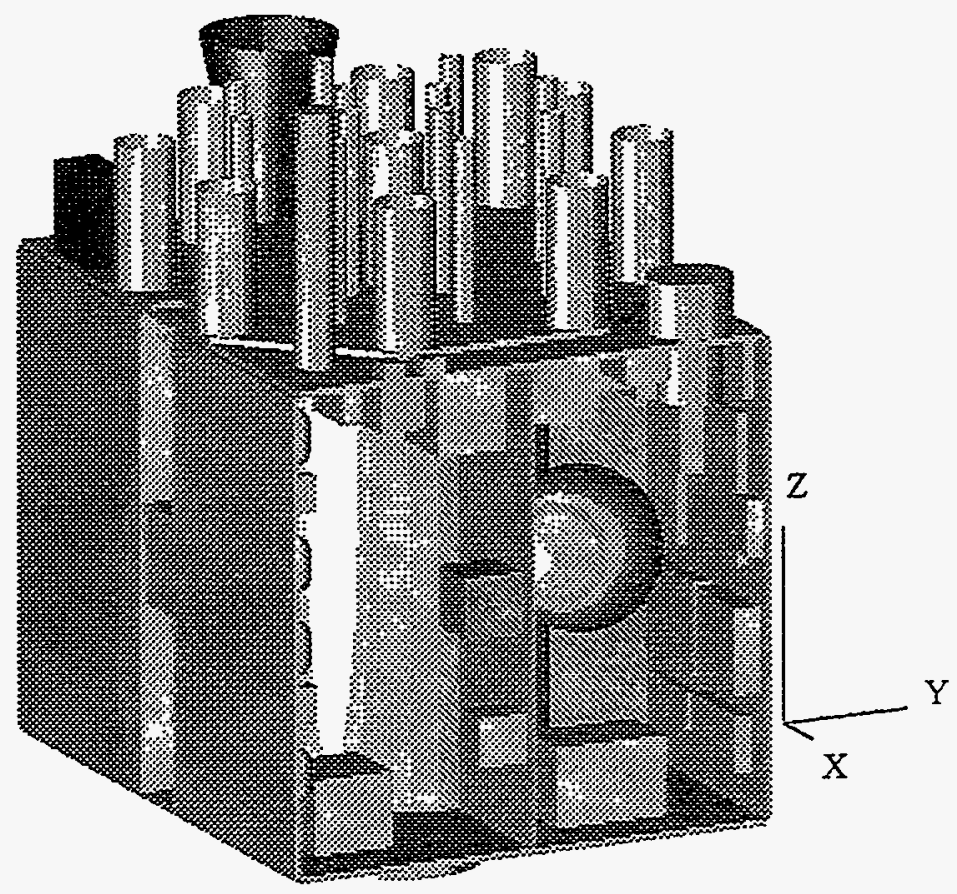

Figure 1. MGED model of GPS Block IIR.

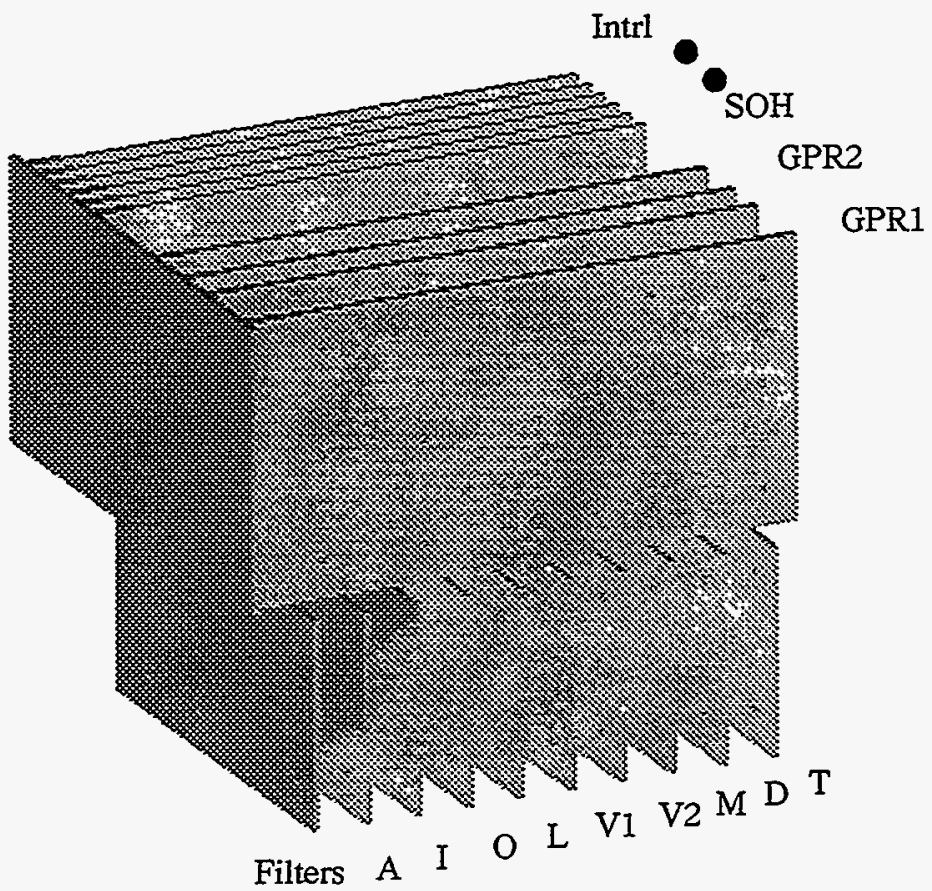

Figure 2. MGED model of single GPS subsystem. 


\section{Using SANDOSE with MGED}

Displaying and Analyzing the Model

MGED allows the modeler to edit/display either all or part of a particular model, depending on how the model has been generated (i.e. the model's "tree"), using the $e$ command. SANDOSE, in turn, provides mass sectoring analysis of the model (or portion thereof) which is currently being edited using the MGED $e$ command. Using this approach, total-dose results can be obtained for either sub-assemblies or the entire model.

With either all or a portion of the model being edited/displayed with the MGED $e$ command, SANDOSE is invoked from within MGED by entering the command sandose. This brings up the GUI as shown in Figure 3. The default settings shown in Figure 3 will be seen the first time the GUI is invoked in an MGED session. Thereafter, if changes are made to the settings, the GUI is quit and then restarted in the same session, the previous changes will be remembered. All of the options available with SANDOSE are selectable on-the-fly by pointing and clicking. Table 1 describes the contents of the GUI. Many of these are self-explanatory, but each will be discussed in the following paragraphs.

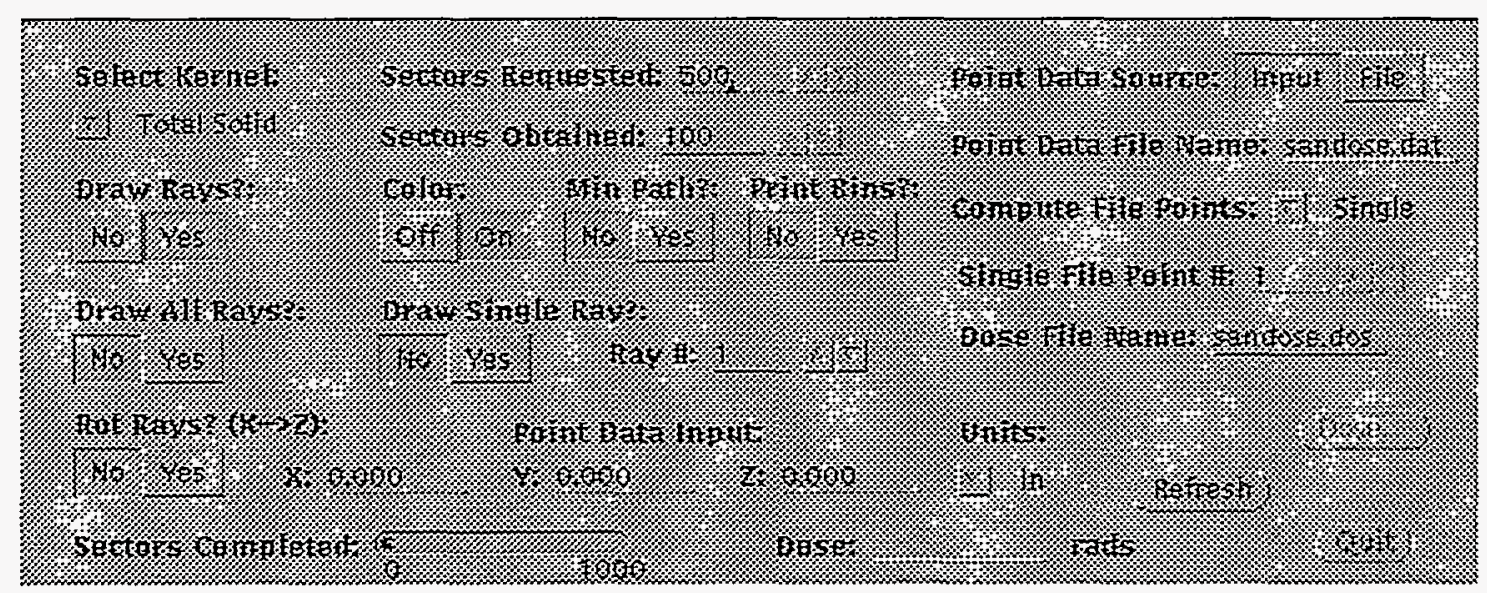

Figure 3. SANDOSE default GUI.

\section{Kernels and Kernel Files}

The kernels presently available include Natural electron, Enhanced electron, and Total electron/proton for GPS Block IIR. The user may also specify a user-defined kernel which must be placed in the file userker. An example user.ker file is shown in Figure 4. Only the $y$-values (dose) are specified in the file. The $x$-values (depth), are specified in SANDOSE at the following 26 depths (in inches):

$$
\begin{aligned}
& 0.005,0.01,0.025,0.05,0.075,0.1,0.125,0.15,0.175,0.2,0.25,0.3,0.35,0.4, \\
& 0.45,0.5,0.55,0.6,0.65,0.7,0.75,0.8,1.0,2.0,5.0,10.0 \text {. }
\end{aligned}
$$




\section{Table 1. Components of the SANDOSE GUI.}

Select Kernel: $\quad$ User selection between available kernels

Sectors Requested: User input of desired number of sectors (typically 500 is adequate)

Sectors Obtained: $\quad$ SANDOSE message of actual number of sectors obtained (not necessarily $=$ request)

Point Data Source:

Input:

File:

Point Data Input:

$X: \quad$ User input of X-coordinate of dose point if "Input" selected

Y: $\quad$ User input of Y-coordinate of dose point if "Input" selected

$Z$ : User input of Z-coordinate of dose point if "Input" selected

Point Data File Name:

Compute File Points: User input of point file name

All:

User input of coordinates in X:, $Y:, Z$ : (see below)

Read coordinates X, Y, Z from point data file

Single:

Single File Point:

Dose File Name:

Rot Rays?:

Min Path?:

Units?:

Color:

Draw Rays?:

Draw All Rays?:

Draw Single Ray?:

Ray Num?:

Print Bins?:

Refresh Button:

Dose Button:

Quit Button:

Sectors Completed:

Dose:

Stream through entire file computing dose at each point

Select a single point from file to compute dose

User input of dose point number from "Point Data File Name"

User input of output file name to contain dose results

Should rays be rotated such that $x->z$ ?

Should minimum path approximation be used near slabs?

Units of dose point locations $\mathrm{X}, \mathrm{Y}, \mathrm{Z}$ (inches or $\mathrm{mm}$ )

Turn off/on color(s) of model so rays drawn over model show up

Should $\mathrm{max} / \mathrm{min}$ rays be drawn over model?

Draw all rays or just max/min areal density rays? (Draw

Rays must be set to Yes to get all rays)

Draw single ray out of all rays requested? (Draw Rays must be set to No to draw single ray)

Ray number of single ray to draw

Print areal density bins after calculation?

Refreshes MGED graphics window

Begin mass sectoring for given dose point(s)

Quit GUI and return to MGED

Message bar following rays as they are thrown

Resulting total dose in rads(Si) 
New kernels can be derived by computing the dose, for the given isotropic radiation environment, at the center of 26 solid aluminum spheres. The radius of each sphere is one of the depth values above. The 26 resulting doses must be placed, in order of increasing depth, in the kernel file userker as shown in Figure 4.

$1 . \mathrm{E}+08$

$9.899 \mathrm{E}+06$

$6.161 \mathrm{E}+06$

$2.776 \mathrm{E}+06$

$1.378 \mathrm{E}+06$

$720.1 \mathrm{E}+03$

$432.7 \mathrm{E}+03$

$263.1 \mathrm{E}+03$

$136.2 \mathrm{E}+03$

$77.89 \mathrm{E}+03$

$33.40 \mathrm{E}+03$

$15.45 \mathrm{E}+03$

$8.103 \mathrm{E}+03$

$5.796 \mathrm{E}+03$

$4.590 \mathrm{E}+03$

$3.990 \mathrm{E}+03$

$3.515 \mathrm{E}+03$

$3.016 \mathrm{E}+03$

$2.798 \mathrm{E}+03$

$2.622 \mathrm{E}+03$

$2.371 \mathrm{E}+03$

$2.034 \mathrm{E}+03$

$1.651 \mathrm{E}+03$

$743.7 \mathrm{E}+00$

101.

14.

Figure 4. Example user.ker file for SANDOSE.

In order for SANDOSE to function properly, the directory from which MGED is launched must contain the kernel files to be used. These include, for GPS Block IIR, the files nat_solid.ker (Natural electron), enh_solid.ker (Enhanced electron), and tot_solid.ker (Total electron/proton). Again, the user defined kernel must reside in the file user.ker. If these files are not available in the launch directory, SANDOSE will display the message: ERROR: Can't open kernel file, and not continue with the analysis.

\section{Sectors}

The maximum number of sectors which can be requested is 5000 , which can be increased by changing one parameter in the source code. Experience has shown that 500 
sectors is adequate to produce good results, since increasing the number above 500 typically changes the result by less than $10 \%$. Since SANDOSE tries to fit the sectors such that each is approximately the same subtended solid angle, the actual number of sectors obtained is often not exactly the number requested.

\section{Dose Point Locations, Dose Output File, and Units}

The source for the dose-point locations can be either from a data file (Point Data Source $=$ File $)$ or input directly into the GUI (Point Data Source $=$ Input). Point Data File Name is the name of the file from which SANDOSE reads the desired dose point locations if File is selected on Point Data Source. An example Point Data File is shown in Figure 5. The first line in the file contains the number of dose points in the file. Each of the following lines contains the $\mathrm{X}, \mathrm{Y}$, and $\mathrm{Z}$ locations of the individual dose points. The maximum number of points in the file is presently set to 500 , but can be changed with a parameter in the code. If Input is selected, points are input one at a time by inserting values into the $X$ :, $Y$, and $Z$ : fields. The units of these dose-point locations can either be in inches or $\mathrm{mm}$, selectable with the Units button.

\section{5}

$35.7025-.86531 .97$

$35.7025-.86530 .57$

$35.7025-.86529 .17$

$35.7025-2.41531 .97$

$35.7025-2.41530 .57$

$35.7025-2.41529 .17$

$35.7025-3.96531 .97$

$35.7025-3.96530 .57$

$35.7025-3.96529 .17$

$35.7025-5.51531 .97$

$35.7025-5.51530 .57$

$35.7025-5.51529 .17$

$35.7025-6.91531 .97$

$35.7025-6.91530 .57$

$35.7025-6.91529 .17$

Figure 5. Example Point Data File for SANDOSE.

If the Point Data Source is File, SANDOSE computes all points in the file if All is selected on Compute File Points, or a single selected point from the file if Single is selected. Single File Point is the number of the single point in the file to be computed. Dose File Name is the name of the output file where SANDOSE writes the results of each dose calculation (The Dose button initiates each calculation). This file is overwritten each time the Dose button is activated, so be careful to save the previous file or enter a new file name for each long series of calculations. SANDOSE writes a single line to the file for each dose point with the $x, y, z$ location of the point and the resulting dose. 


\section{Ray Rotation}

When SANDOSE computes the appropriate directions of rays to cover the entire $4 \pi$ steradians with equal solid angle per sector, it always places the first ray up the $z$-axis, and fills rings in azimuth to fill in the upper half space. Often, a ring of rays is placed in the $x y$ plane at $\mathrm{z}=$ const. This is normally not an issue unless the model happens to be a series of slabs representing closely spaced circuit boards. If the boards lay in xy planes and a dose point is placed between the boards, that ring of rays in the xy plane will exit the entire system of boards without intersecting anything. Experience has shown that this situation tends to give a significant overestimate of the total dose at such a dose point. To alleviate this, capability exists to rotate all rays such that $x->z$ (and $z->-x$ ) in order to avoid this situation when circuit boards lay in xy planes. Rot Rays determines whether the ray distribution is rotated or not.

\section{Min Path Approximation}

A minimum path approximation can be applied by using the Min Path option. The purpose of minimum path is to get the worst-case approximation to actual radiation transport. With this option, any time a ray penetrates a wall at a non-normal angle of incidence, the penetration depth is taken to be the thickness of the wall rather than the path length, which is longer and therefore provides more areal density. If this option is selected, SANDOSE presently only applies the minimum path if the angle between the surface normal and the ray is greater than 60 degrees, which effectively requires the dose point to be very close to the wall before minimum path is applied. SANDOSE also checks that both entry and exit surfaces of the wall are parallel before applying minimum path, thereby attempting to avoid applying it to corner crossings and other undesirable instances. Obviously, if Min Path is activated, a larger total dose prediction will be obtained. This minimum-path approximation is experimental and should be used accordingly.

\section{Graphical Display of Dose Contribution per Sector}

Graphical display of the relative dose contribution per sector for the model gives the user a qualitative feel for where most of the dose to a particular point comes from. It can also help determine where spot shielding should be added to reduce the total dose at a particular point. For example, a relatively small solid angle may be revealed which provides most of the dose. If Draw Rays is Yes and Draw All Rays is No, only the maximum and minimum areal density rays (minimum and maximum dose rays) are drawn, the minimum dose ray in blue and the maximum in red. The values of these are shown in the upper right corner of the graphics window, and also in the text window. If Draw All Rays is also Yes, all rays are drawn using a color scheme with the lowest dose contributors in blue up to the highest in red. A table of these colors and sector dose values is again shown in the upper right corner. A single selected ray can also be drawn if Draw Single Ray is Yes, Draw Rays is Yes, and Draw All Rays is No. These ray-display options are meant primarily to give a qualitative feel for where most of the total-dose contribution comes from to compare with intuition. 


\section{Areal Density Bins}

Areal density bins provide an indication of how the total dose is accumulated over the sectors and how much shadow shielding is being provided. For example, it may be that just a few unshielded sectors produce $50 \%$ of the total dose. SANDOSE areal density bins can be printed by setting Print Bins to Yes. The areal density spectrum is divided into 20 bins and each sector's contribution is placed into the appropriate bin during the calculation.

\section{Refresh, Dose, and Quit}

The Refresh button simply refreshes the MGED graphics window from within SANDOSE in order to remove drawn rays from a previous mass-sectoring calculation. This happens automatically between calculations as well. The Dose button starts a mass-sectoring calculation, and the Quit button exits SANDOSE and returns to MGED.

\section{Example SANDOSE Results}

Figure 6 shows an example of total-dose results for the subsystem shown in Figure 2. These results are for 500 sectors per dose point using the Total electron/proton kernel with ray rotation turned off. Depending on the complexity of the model, a typical dose point takes from 0.5 to 5 seconds to complete on a SUN SPARCstation 2. For this particular subsystem on the GPS Block IIR model, each point requires approximately 4 seconds. The boards in this subsystem model contain only 15 dose points per side. Results on individual boards are interpolated by the plotting package from the individual dose points.

Figure 7 gives the exact SANDOSE results for Board GPR1, sides 1 and 2, of this particular subsystem. This is the board labeled GPR1 in Figure 2. The first three columns are the location of the dose point and the fourth column is the dose, in rads ( $\mathrm{Si}$ ). These results can be used to verify the SANDOSE installation.

\section{Accuracy and Sources of Error}

\section{General Accuracy Requirements}

Since mass sectoring is known to be a crude but efficient approximation to rigorous radiation transport techniques, SANDOSE results are expected to be in error, sometimes significantly. The benchmark results discussed below have, in almost all cases, shown this error to be a moderate overestimate of the dose. Therefore, a conservative result is obtained, adding slightly to the required design margin. This is the key point which makes the mass-sectoring technique in general, and SANDOSE in particular, useful. If the code can be relied upon not to underestimate the dose, then the user can feel confident that egregious errors will not be made. On the other hand, excessive overestimation of the dose can be detrimental by adding unnecessary cost to the system. As described below, SANDOSE strikes a reasonable balance between these extremes. 
(This page intentionally left blank) 


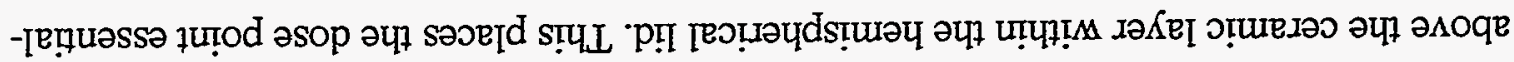

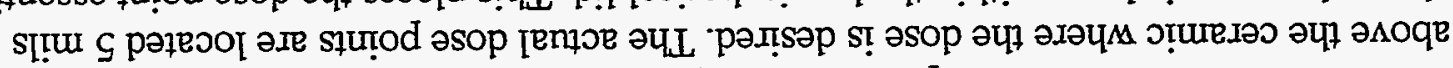

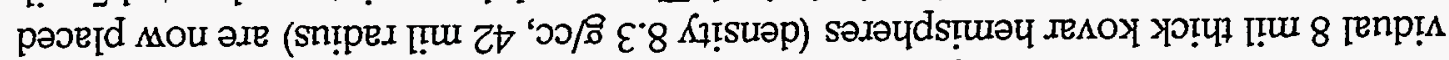

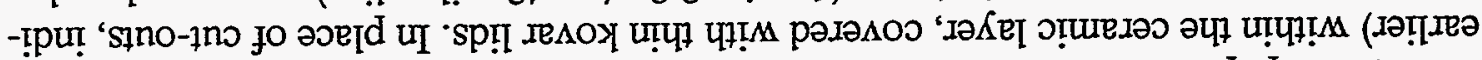

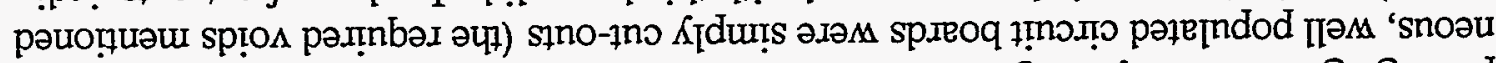

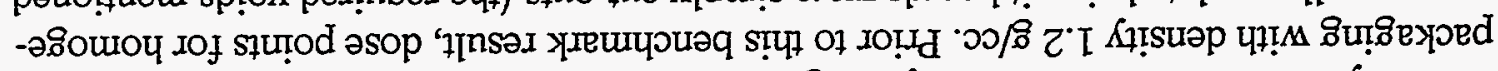

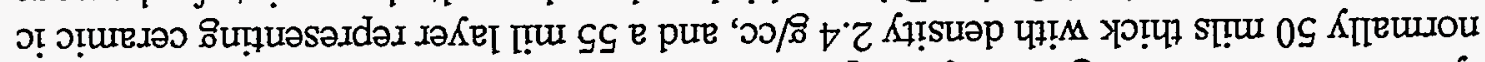

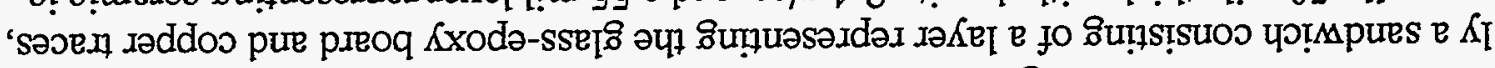

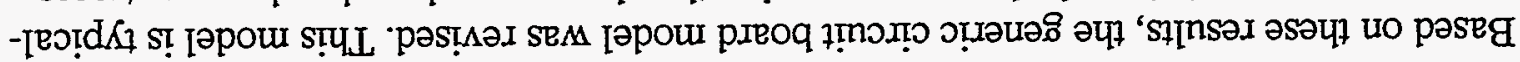

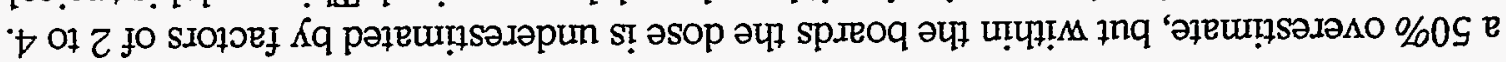

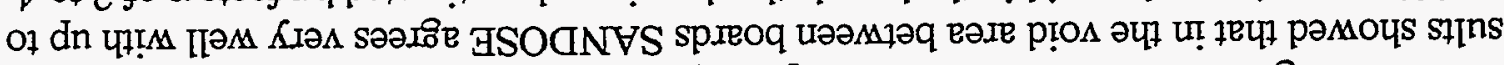

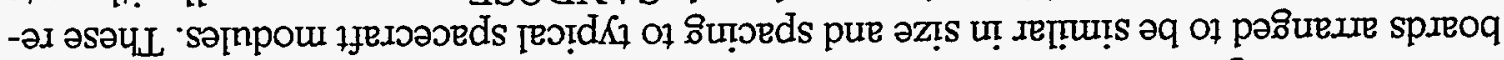

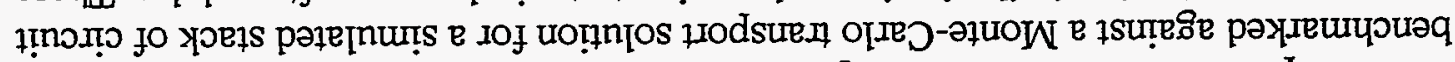

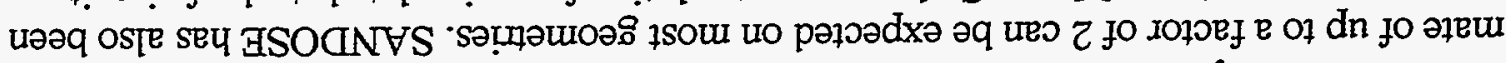

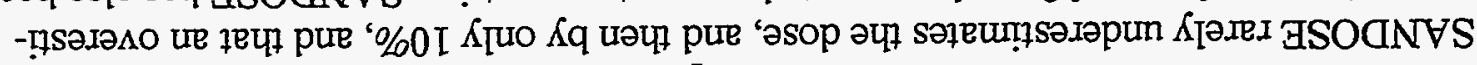

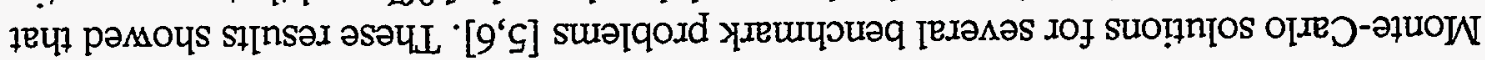

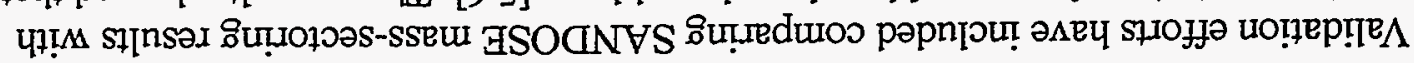

\section{uo!ıep!e^ $\exists S O A N \forall S$}

\section{¿}

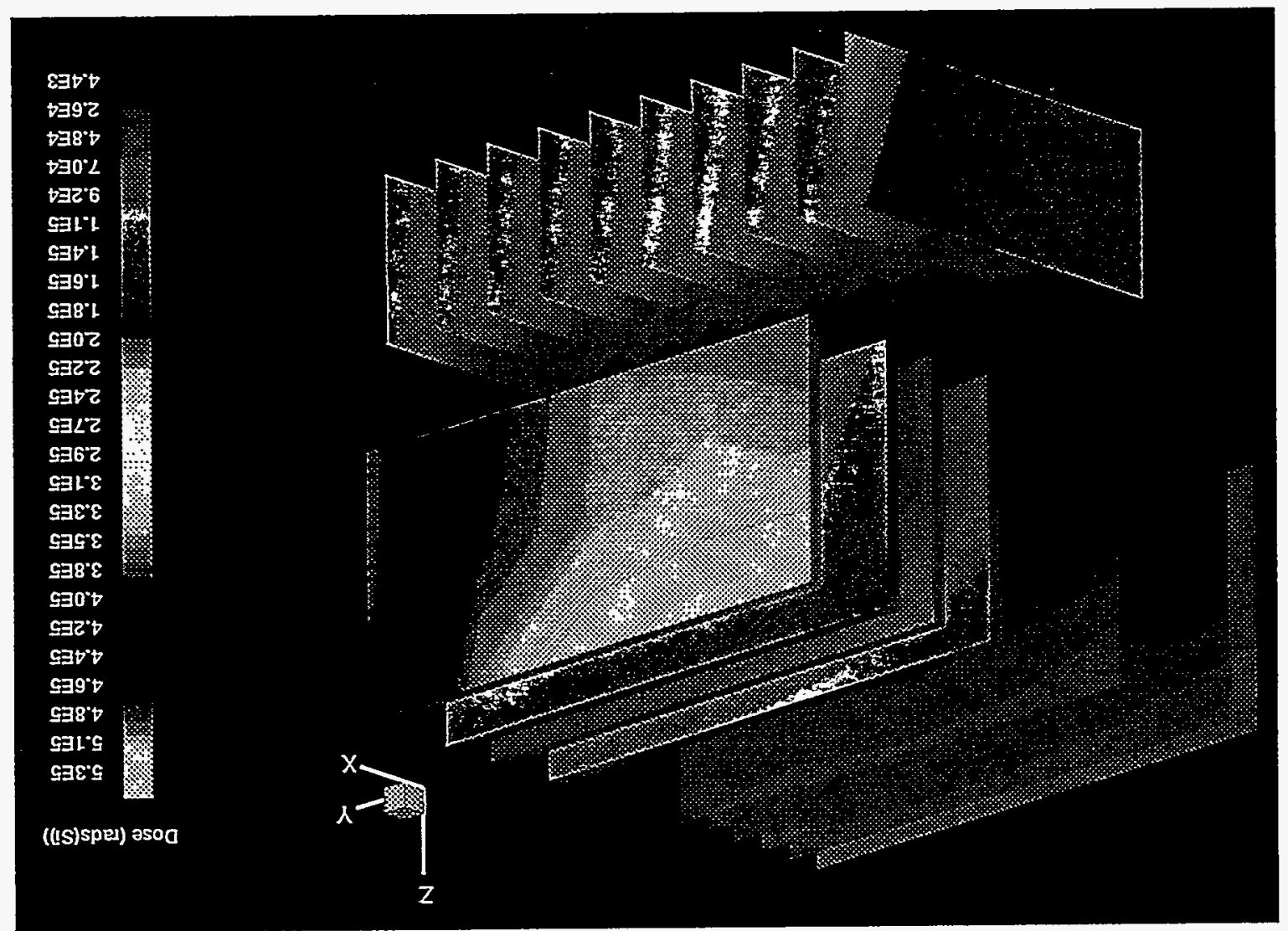


ly between the boards, so the region "within" the board where the mass-sectoring method clearly ignores too much physics is avoided. Examples of these generic circuit board models can be found in the GPS Block IIR model included in the distribution (gpsrev2g.g).

Based on these limited validations, SANDOSE results most likely fall somewhere between $90 \%$ of actual (10\% underestimate) and $200 \%$ of actual (x2 overestimate). This kind of error is an acceptable trade-off considering the efficiency of the technique and the difficulty of obtaining rigorous 3-D transport solutions. It should be recognized, however, that the benchmark database accumulated so far is very limited and it is possible that other problems might produce results outside of these bounds. It has been our policy at Sandia that as the system design begins to be finalized, SANDOSE results are further validated with appropriate rigorous Monte-Carlo calculations.

$35.7025-0.86531 .97396588$

$35.7025-0.86530 .57373140$

$35.7025-0.86529 .17368102$

$35.7025-2.41531 .97503591$

$35.7025-2.41530 .57476799$

$35.7025-2.41529 .17469397$

$35.7025-3.96531 .97536376$

$35.7025-3.96530 .57503070$

$35.7025-3.96529 .17501604$

$35.7025-5.51531 .97538873$

$35.7025-5.51530 .57509940$

$35.7025-5.51529 .17499252$

$35.7025-6.91531 .97542637$

$35.7025-6.91530 .57505834$

$35.7025-6.91529 .17494927$

$35.5325-0.86531 .9735216 .3$

$35.5325-0.86530 .5727672 .8$

$35.5325-0.86529 .1721705$

$35.5325-2.41531 .9736132 .6$

$35.5325-2.41530 .5727940$

$35.5325-2.41529 .1721898 .8$

$35.5325-3.96531 .9731231 .6$

$35.5325-3.96530 .5726411 .3$

$35.5325-3.96529 .1723256$

$35.5325-5.51531 .9736557$

$35.5325-5.51530 .5729963 .8$

$35.5325-5.51529 .1728455 .7$

$35.5325-6.91531 .9749362$

$35.5325-6.91530 .5734767 .7$

$35.5325-6.91529 .1734005 .3$

Figure 7. Exact SANDOSE results for Board GPR1, Sides1 and 2. 


\section{Additional Sources of Error}

Other possible errors which must be considered are the accuracy of the environment specification and the accuracy of the BRL-CAD model. For spacecraft, the space radiation environments are typically obtained from available proton and electron models and must be appropriate for the orbit of interest. As mentioned earlier, the required model accuracy decreases as the distance from the dose point increases. It is ultimately up to the user how much time and effort he or she is willing to invest in the modeling. Adding large amounts of detail is probably only detrimental in that the calculation time will increase with the complexity of the model. During ray tracing, modeling errors in the form of overlaps or interference between objects are detected and a message is issued from BRL-CAD each time a ray intersects objects which occupy the same space. In this case, the modeler must correct the model to eliminate such errors.

\section{Incorporating SANDOSE into BRL-CAD}

Since SANDOSE has been incorporated into the BRL-CAD model editor MGED, installing the module into an existing BRL-CAD site requires some user familiarity with the organization of BRL-CAD, and especially MGED. SANDOSE can either be added to an existing site as described below or the entire brlcad/mged subdirectory can be replaced from the SANDOSE distribution if the BRL-CAD versions agree.

The addition of the SANDOSE module to MGED involves adding the SANDOSE modules sandose.c, sandose_ui.c, and the include file sandose_ui.h to the mged subdirectory. Changes are also required to some of the MGED modules, including attach.c, buttons.c, cmd.c, and edsol.c. The MGED include files dm.h, ged.h, and sedit.h also require changes. The software distribution includes a complete brlcad/mged subdirectory with all of these changes for BRL-CAD Release 4.0.

An enhanced version of the MGED X-window driver, dm-X.c, is also included in the distribution. This driver has been improved to provide color display and to include a new function for SANDOSE to draw color lines, X_2d_color_line(). At least a hook for this new function must also be added to all of the other $\mathrm{dm}$-* driver modules which are being used in the site build. Other driver modules included in the distribution which have been updated with this function hook include dm-plot.c, dm-ps.c, dm-tek.c, and dm-tek4109.c. (The addition of this hook is only to allow the code to build properly. This distribution of SANDOSE only supports $X$-windows graphics.) Other driver modules may also need to have this hook added depending on which displays are enabled at the particular site. If it is desired to actually use one of the display drivers other than $\mathrm{X}$, then the code for the function *_2d_color_line ( ) must be written and installed in the appropriate driver module.

In order for MGED to build properly, the file Cakefile must have sandose.c and sandose_ui.c added to the list of modules for mged. The new Cakefile is also included in the distribution. Assuming that all of this is in place, the user simply uses cake (a super make utility included with BRL-CAD) to build the new MGED executable. Simply cd to the brlcad/mged subdirectory, type cake, and MGED is built. 


\section{Availability}

SANDOSE is available via license by contacting either the Sandia National Laboratories Technology Transfer Center or the author. The distribution includes source code for SANDOSE, example kernel files, an example MGED model of a satellite similar to Figure 1 , and a point data file for the subsystem in Figure 2. These and the results in Figures 6 and 7 can be used to verify the installation.

\section{Conclusions}

SANDOSE is a useful tool for estimating total dose to a BRL-CAD model due to isotropic radiation. The code is based on mass sectoring, which applies a 1-D shadow-shielding approach to 3-D geometries. Errors to be expected from such an approximation range from a slight $(10 \%)$ underestimate to a factor of 2 overestimate of the dose. The nature of the mass-sectoring approximation allows a large amount of model simplification, through homogeneous piece-part modeling away from the dose points, without greatly effecting the results. SANDOSE contains graphical diagnostic tools to qualitatively examine the model to determine where most of the shadow shielding is for a given dose point. This allows the modeler to apply spot shielding, if necessary, to reduce the dose at that point and then view the results graphically.

\section{References}

[1] BRL-CAD Release 4.0 manual, Advanced Computing Systems, The U. S. Army Ballistic Research Laboratory, Aberdeen Proving Ground, Maryland, 21005-5066.

[2] N. Smith, "Satellite Vulnerability Codes User's Manual," WL-TR-90-70, AFSC WL, Nov. 1990.

[3] W. R. Yucker, "SIGMA: Space Radiation Dose Analysis Within Complex Configurations," RSIC Computer Code Collection CCC-118, May 1974.

[4] L. J. Lorence, Jr., J. E. Morel, and G. D. Valdez, "User's Guide to CEPXS/ONEDANT: A One-Dimensional Coupled Electron-Photon Discrete Ordinates Code Package," Sandia National Laboratories Report SAND89-1661, Sep. 1989.

[5] Memo dated February 11, 1992, C. D. Turner and S. B. Roeske, 9352, to J. F. Hudson, 9212, Subject: Mass Sectoring Analysis of the W-Sensor on GPS

[6] Ronald Saqui, et al, "Computational Benchmarks for Electron Total Dose Shielding Methodology,” WL-TR-90-59, AFSC WL, Jan. 1991. 


\section{DISTRIBUTION:}

1 MS 1083 P. S. Winokur (1332)

1 MS 0971 G. H. Mauth (9203)

1 MS 0972 J.P. Kern (9205)

1 MS 0967 L. W. Maschoff (9206)

1 MS 1166 J. H. Renken (9301)

1 MS 1179 T. F. Wrobel (9341)

1 MS 1167 E. F. Hartman (9351)

1 MS 1167 L. D. Posey (9351)

1 MS 1166 G. J. Scrivner (9352)

1 MS 1166 S. B. Roeske (9352)

20 MS 1166 C. D. Turner (9352)

1 MS 0357 M. R. Ackermann (9835)

1 MS 9018 Central Technical Files (8523-2)

5 MS 0899 Technical Library (13414)

1 MS 0619 Technical Publications (12613)

2 MS 0100 Document Processing for DOE/OSTI (7613-2) 